\title{
Rotation and Line Broadening in OBA Stars
}

\author{
Ian D. Howarth \\ Dept. Physics \& Astronomy, UCL, Gower St., London WC1E 6BT, UK
}

\begin{abstract}
Observational $v \sin i$ line-width measurements for OBA stars are summarized, and the relationship of these measurements to $v_{\mathrm{e}} \sin i$, the true projected equatorial rotation velocity, reviewed. In general, $v_{\mathrm{e}} \sin i \geq v \sin i$ for Be stars, while $v_{\mathrm{e}} \sin i \leq v \sin i$ for O-type stars. The implications are that (i) it is probable that $\mathrm{Be}$ stars are closer to their critical rotation velocities than is generally supposed (rendering plausible a range of new physical mechanisms for the Be phenomenon); and (ii) some non-rotational macroscopic line broadening ('turbulence') occurs in the atmospheres of O stars (and BA supergiants). Physical interpretations of this 'turbulence' are considered.
\end{abstract}

\section{Introduction}

An overview of determinations of the line-width parameter $v \sin i$ for early-type stars is given in Fig. 1. General points to note are that Be stars are, as a class, more rapid rotators than B-normal main-sequence stars; and that there is an almost complete absence of narrow-lined spectra among Galactic O stars (of all luminosity classes), and among early-type supergiants in general.

\section{Calibrations}

Almost all large-scale surveys of the $v \sin i$ parameter in OBA stars are based on one of two approaches (cf. Table 1). The first is to infer $v \sin i$ from line width at half depth, calibrating against the system of rotational standards set up by Slettebak et al. (1975). The second is to compare observations with 'spun-up' versions of narrow-line stars, or model-atmosphere flux spectra (either directly, or through construction of an intermediate $v_{\mathrm{e}} \sin i$-line-width calibration), where the 'spin-up' is achieved through a convolution with a rotational-broadening function (cf. Gray 1992).

In practice, both approaches assume a single-valued (usually linear) correspondence between line width and the projected equatorial rotation velocity, $v_{\mathrm{e}} \sin i$. However, in general there is no such single-valued correspondence; Fig. 2 provides an illustration of this, and demonstrates that a spread of order $\sim 10 \%$ in $v_{\mathrm{e}} \sin i$ is easily obtained at fixed line width. This is because not only $v_{\mathrm{e}} \sin i$, but also the axial inclination and, most importantly, the ratio $v_{\mathrm{e}} / v_{\mathrm{c}}$ play a role in line width (where $v_{c}$ is the equatorial velocity at which the effective gravity is zero). Furthermore, for the most rapid rotators, there is an increasing redundancy between line width and $v_{\mathrm{e}} \sin i$ (e.g., Stoeckley 1968; Townsend, Owocki \& Howarth, in prep.), and this has significant implications for Be stars (§3). 


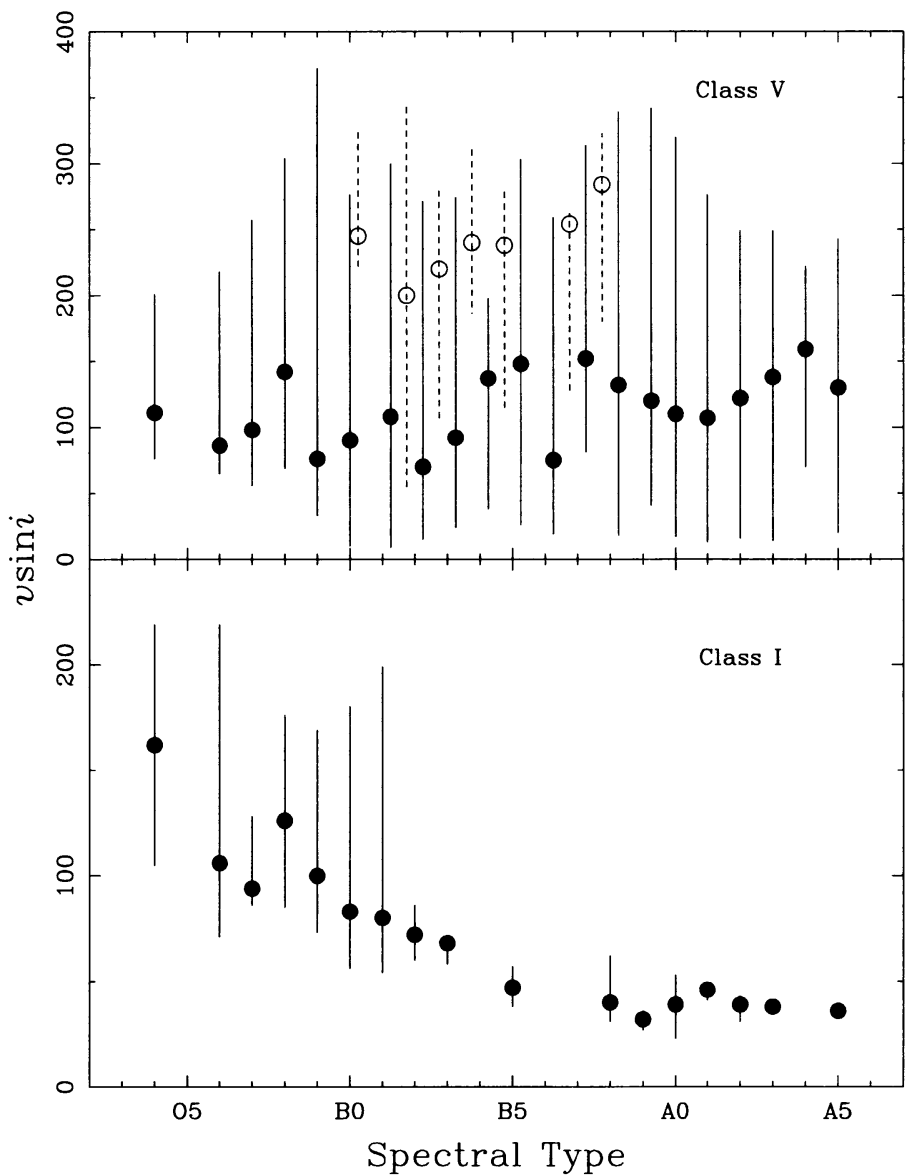

Figure 1. Distribution of $v \sin i-$ n.b., not $v_{\mathrm{e}} \sin i$ - for Galactic early-type stars (distilled from Howarth et al. 1997, Howarth \& Siebert in prep., Verdugo et al. 1999, Royer et al. 2002). Circles show median values as a function of spectral type (Be stars as open symbols), and vertical bars show total ranges, but with the extreme values clipped. Note the different vertical scales used for luminosity class I and class V stars.

\subsection{The Slettebak system}

The benchmark against which observers habitually calibrate or compare their results is the Slettebak system (Slettebak et al 1975, hereinafter SC75). SC75 established this system of rotational 'standard stars' by comparing their digital data with numerical models. The models were constructed by adopting Roche geometry, uniform angular velocity at the surface, von Zeipel gravity darkening (justified observationally), and numerical integration of angle-dependent modelatmosphere intensities (Collins 1974). The approach was thorough and, barring technical improvements, is what one would do today if starting from scratch; the influence of this seminal paper can be seen from inspection of Table 1 .

Although SC75 did include some rapid rotators, a much larger sample of fast-rotating Be and shell stars was surveyed by Slettebak (1982; S82). However, 


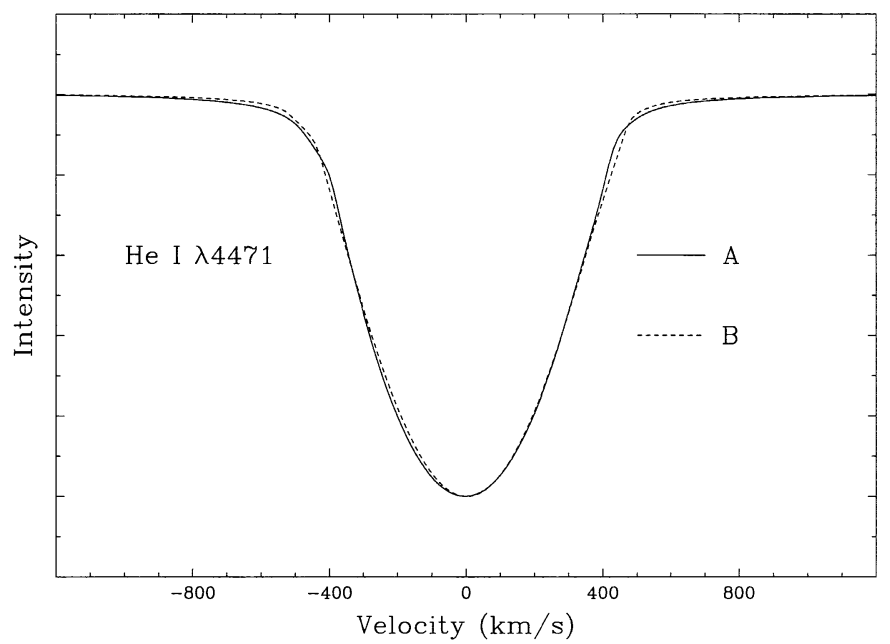

Figure 2. Two models of the He I $\lambda 4471$ line. Each model has $i=90^{\circ}$, $T_{\text {eff }}=27.3 \mathrm{kK}$, and identical full-width at half depth. However, model 'A' has $v_{\mathrm{e}} \sin i=450 \mathrm{~km} \mathrm{~s}^{-1}\left(\omega / \omega_{\mathrm{c}}=0.95\right)$ while model 'B' has $v_{\mathrm{e}} \sin i=406 \mathrm{~km} \mathrm{~s}^{-1}$ $\left(\omega / \omega_{\mathrm{c}}=0.05\right)$. Although model B is physically unrealistic, this does illustrates that (i) $v_{\mathrm{e}} \sin i$ is not a single-valued function of line width, and (ii) models that do not take into account gravity darkening (etc.), such as model B, will underestimate $v_{\mathrm{e}} \sin i$.

instead of comparision with numerical models, the technique used in S82 was "visual comparison of line widths on the [photographic] spectrograms with those of standard rotational-velocity stars" from SC75 (such eye-estimates being the standard technique at that time).

As a basis for $v_{\mathrm{e}} \sin i$ calibrations, the Slettebak system therefore has some generally unrecognised limitations. First, the methodology for the high- $v \sin i$ secondary standards leaves room to question the objectivity, accuracy, and precision of the results, notwithstanding the quality of Slettebak's observational material and his undoubted skill as an observer. Secondly, any practical application of the Slettebak system necessarily assumes a single-valued correspondence between $v_{\mathrm{e}} \sin i$ and line width; as we have seen, this assumption is ill-founded. Furthermore, the model results illustrated by Collins (1974), which underpin the Slettebak system, show increasing line width with increasing $\omega / \omega_{\mathrm{c}}$ (at fixed $v_{\mathrm{e}} \sin i$ ). This behaviour is difficult to understand physically, and is not reproduced by more-recent models.

\section{2. 'Spun-up' models}

Models generated by rotational convolution of narrow-lined standards don't take into account any of the physical effects included in Roche-based models. As well as neglecting the effect of the change in limb-darkening through the line, a convolution therefore corresponds to the assumption that $\omega / \omega_{\mathrm{c}} \equiv 0$ (at any $\left.v_{\mathrm{e}} \sin i\right)$ - perhaps acceptable for small to moderate rotation, but increasingly inaccurate for $\omega / \omega_{\mathrm{c}} \gtrsim 0.5$. This assumption always results in model line-widths that are too large for the $v_{\mathrm{e}} \sin i$ (cf. Fig. 2), because in nature the fast-rotating, gravity-darkened equatorial regions make a smaller flux contribution to the line 
Table 1. Some large-scale surveys of $v \sin i$ for OBA stars. The calibrations are with respect to Slettebak et al. 1975 and Slettebak 1982 (SC75, S82); by convolved, or 'spun-up', models (C); or by analysis of the Fourier transforms $(\mathrm{F})$, which is the frequency-space equivalent of using spun-up models. 'Vis' means visual estimates from photographic plates, rather than measurements)

\begin{tabular}{lcl}
\hline \multicolumn{1}{c}{ Author } & Types & Calibration \\
\hline Balona (1975) & B & SC75 \\
Conti \& Ebbets (1977) & O & C+SC75-vis \\
Wolff et al. (1982) & B & SC75-vis \\
Abt \& Morrell (1995) & A & SC75 \\
Halbedel (1996) & B, Be & SC75, S82 \\
Penny (1996) & O & C (via CE77) \\
Howarth et al. (1997) & OB & C \\
Steele et al. (1999) & Be & SC75 \\
Chauville et al. (2001) & Be & C \\
Royer et al. (2002) & A & F \\
Abt, Levato \& Grosso (2002) & B & SC75 \\
\hline \hline
\end{tabular}

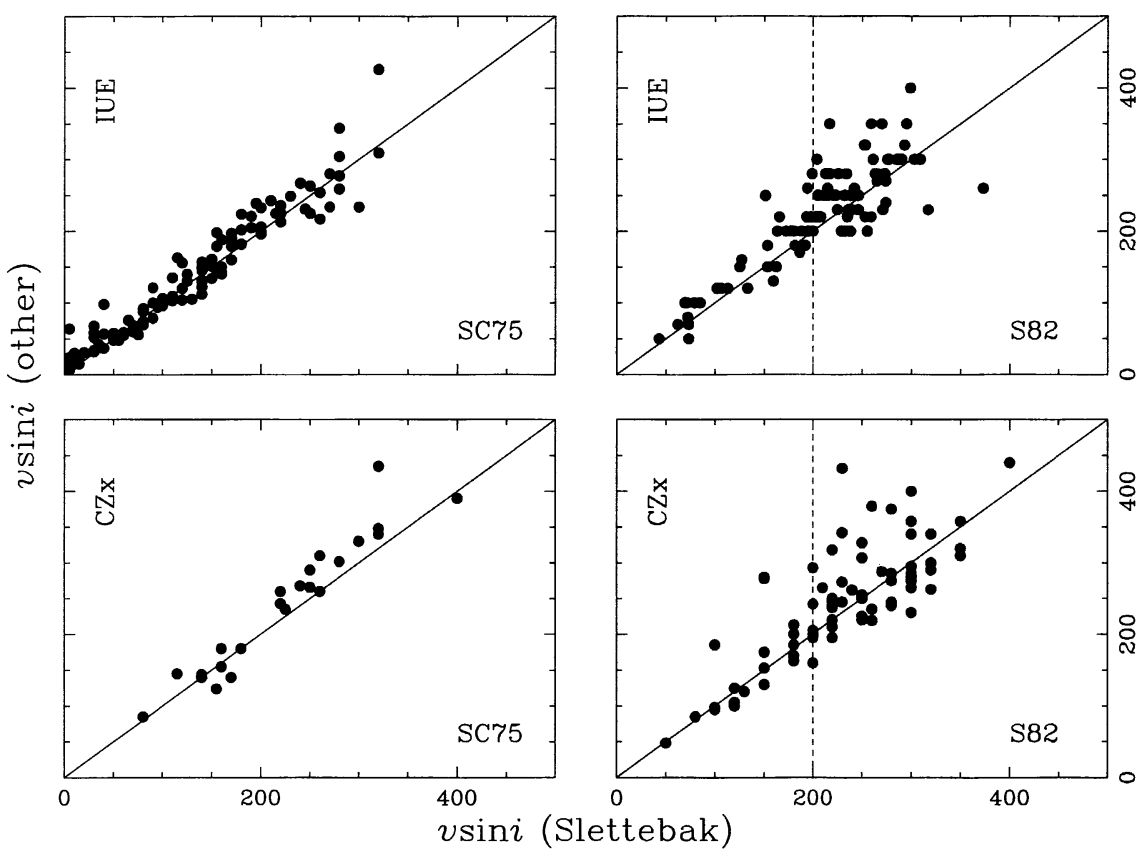

Figure 3. Comparison of B-star $v \sin i$ results from SC75 (left) and S82 (right) with preliminary results from Howarth \& Siebert (in prep., upper) and Chauville et al. (2001, lower). The last two sources use 'spun-up' models for calibration. Note the large scatter compared to the S82 results, especially for $v \sin i \gtrsim 200 \mathrm{~km} \mathrm{~s}^{-1}$. 
profiles than a convolution assumes. In other words, spun-up models always lead to an underestimate of $v_{\mathrm{e}} \sin i$.

The factors contributing to this difference include geometry (spherical vs. Roche); treatment of limb darkening (constant vs. wavelength-dependent); variation of line strength with latitude; and variation of continuum intensity with latitude. For most of the parameter space applicable to real stars, the first two aspects are of relatively minor importance. The role of line-strength variation with latitude (i.e., temperature) depends on the specific feature under consideration; but, in general, the most important factor is the variation in continuum strength with latitude. For example, the equivalent widths of He I $\lambda 4471$ and Mg II $\lambda 4481$ (the lines most commonly used in B-star $v \sin i$ determinations) vary by less than $\sim 20 \%$ going from $30 \mathrm{kK}$ to $20 \mathrm{kK}$ ( $\sim \mathrm{B} 0$ to B2.5), while the $\sim 440 \mathrm{~nm}$ continuum level drops by a factor $\sim 2$ over the same range. It is, therefore, the von Zeipel effect that makes fast-rotating equatorial regions difficult to detect. It is also why increasing $v_{\mathrm{e}} \sin i$ has little effect on model line widths as $v_{\mathrm{c}}$ is approached.

\section{Be stars}

It has long been known that Be stars are, as a class, rapid rotators (e.g., Struve 1931; Slettebak 1966), and all important aspects of a Be episode can be broadly understood in terms of ejection of material in the equatorial plane. The data in Fig. 1 imply that, typically, $v \sin i / v_{\mathrm{c}} \geq 0.5\left(\omega \sin i / \omega_{\mathrm{c}} \simeq 0.7\right)$ - on the Slettebak system. However, if published $v \sin i$ values for Be stars were systematically smaller than $v_{\mathrm{e}} \sin i$, as suggested above, then the effective equatorial gravity would be closer to zero than generally supposed. This in turn would make the ejection of material into the circumstellar environment much easier, rendering plausible a range of new possibilities for the Be mechanism (Owocki 2003).

\subsection{Rotation}

Fig. 3 compares Slettebak's results with two large surveys calibrated with 'spunup' models (and therefore independent of the Slettebak system). There is generally good agreement with the numerical results of $\mathrm{SC75}$, but the scatter compared to the eye estimates of S82 is very large, especially for $v \sin i \gtrsim 200 \mathrm{~km} \mathrm{~s}^{-1}$. Given the role of S82 in calibrating other catalogues, it seems likely that $v \sin i$ results in the literature are, in general, subject to large $(\not 20 \%)$ random errors, at least for broad-lined (i.e., Be) stars.

The possibility of systematic errors is more important where equatorial mass ejection is concerned. Fig. 3 shows that there is a modest tendency for 'spun-up' results to yield larger $v \sin i$ values than the S82 (and, to a lesser extent, SC75) standards; and yet we know that the 'spun-up' models themselves systematically underestimate $v_{\mathrm{e}} \sin i$. Moreover, line widths become extremely insensitive to $v_{\mathrm{e}} \sin i$ at large rotational velocities, and may even decrease with increasing $v_{\mathrm{e}} \sin i$, as shown by numerous modellers (Stoeckley 1968; Collins \& Truax 1995; Townsend et al., in prep.). This insensitivity, even when noted, has been neglected by observers, who invariably assume a more or less linear relationship between line width and $v \sin i$, regardless of the method of calibration (see references in Table 1), consequently underestimating $v_{\mathrm{e}} \sin i$. 


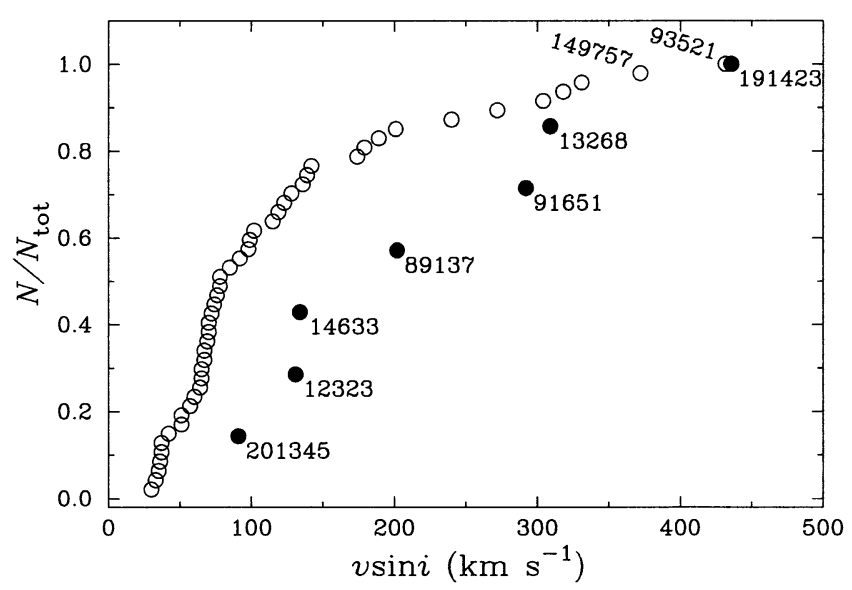

Figure 4. The cumulative distribution of $v \sin i$ for $\mathrm{ON} \mathrm{V}$ and morphologically normally O V stars (filled and open circles, respectively); selected objects are labelled by HD number. The distributions are significantly different (with $97 \%$ confidence), showing that ON spectral morphology is associated with faster-than-average rotation (Howarth \& Smith 2001).

We conclude that, most probably, $v \sin i<v_{\mathrm{e}} \sin i$ for measurements of rapid rotators calibrated against the Slettebak system, as well as those calibrated using spun-up models. The only way to determine reliable $v_{\mathrm{e}} \sin i$ values for Be stars (and other very rapid rotators) is to model directly the line profiles for $v_{\mathrm{e}} \sin i, i$, and $\omega / \omega_{\mathrm{c}}$, using Roche models and accounting for von Zeipel darkening. It is salutory to note how few observational studies have actually taken this approach (e.g., SC75, Howarth \& Smith 2001).

\subsection{Other factors}

In considering the true equatorial effective gravity (and candidates for the $\mathrm{Be}$ mechanism), we should recall that it is not only the accurate measurement of $v_{\mathrm{e}} \sin i$ that is important; the ratio $v_{\mathrm{e}} \sin i / v_{\mathrm{c}}$ also depends on the stellar mass and (e.g., polar) radius. These fundamental parameters are typically obtained from look-up tables as a function of spectral type, rather than being direct determinations. Because there is every reason to suppose that mass and radius are functions of rotation as well as spectral type (cf. Maeder 2003) - and recalling that spectral type is a function of inclination for rapid rotators - this gives further latitude for driving $v_{\mathrm{e}} \sin i / v_{\mathrm{c}}$ closer to unity than has heretofore been generally supposed.

Finally, although a difficulty in some respects, the sensitivity of line profiles to $v_{\mathrm{e}} \sin i / v_{\mathrm{c}}$ and $i$, in addition to $v_{\mathrm{e}} \sin i$, affords a purely spectroscopic method for determination of stellar mass, radius, and distance, independent of any external calibration, because of the different $[M, R]$ dependences of the observable quantities $\log g$ and $v_{\mathrm{e}} \sin i / v_{\mathrm{c}}$. Unfortunately, in practice this 'sensitivity' of the profiles is not very strong, but it can nonetheless be applied to obtain useful results (Howarth \& Smith 2001). 


\section{O-type stars}

The importance of rotation for massive stars, in particular, is that it clearly plays a crucial role in their evolution. Even with ad hoc adjustments such as convective overshoot, traditional evolutionary models didn't incorporate sufficient mixing to introduce CNO-processed material at the stellar surface during core hydrogen burning; the introduction of rotation introduces the required mixing.

The evidence for CNO-processed material at the surface includes the 'helium discrepancy' (excess surface helium abundances; Herrero et al. 1992), the existence of OBN stars (Walborn 1976, Schönberner et al. 1988) and enhanced $\mathrm{N} / \mathrm{H}$ abundances in A-type supergiants (Venn 1999), and boron depletion in B stars (Fliegner, Langer \& Venn 1996). Many of these results rely on physically incomplete atmosphere/radiative-transfer models, but Fig. 4 presents a virtually model-independent demonstration of the importance of rotation to mixing: as a class, ON V stars (whose spectra show enhanced N/C line ratios indicating, most probably, CNO-enhanced abundance ratios) have systematically larger $v \sin i$ values than their spectroscopically normal counterparts.

O-type stars are, as a class, rapid rotators; as illustrated in Fig. 1, there are essentially no known narrow-lined Galactic O-type stars. The range in $v \sin i$ for $\mathrm{O}$ supergiants is significantly narrower than for main-sequence stars, with the smallest $v \sin i$ for any O I star in the Howarth et al. (1997) catalogue being $65 \mathrm{~km} \mathrm{~s}^{-1}$, for the peculiar star HD 105056. There are, however, numerous $\mathrm{O} \mathrm{V}$ stars with smaller $v \sin i$ values (though all are still $\geq 30 \mathrm{~km} \mathrm{~s}^{-1}$ ). It is straightforward to understand how rotation rates may decrease in evolving from type $\mathrm{V}$ to type I, but difficult to imagine how the reverse situation may arise for single stars. The conclusion is that there is some non-rotational, macroscopic line broadening mechanism operating in O-type supergiants (and also BA supergiants, and $\mathrm{O}$ main-sequence stars). The line profiles themselves also demonstrate very clearly the need for a macroscopic broadening mechanism other than rotation, usually being more ' $\mathrm{V}$ '-shaped than the bowl-shaped profile resulting from pure rotation (Fig. 5).

This broadening mechanism was labelled 'turbulence' by Conti \& Ebbets (1977) and by Howarth et al. (1997), although Slettebak (1956) had also alluded to "large-scale atmospheric motions. . .as broadening agents", and even before that Struve (1952) had stated "I strongly suspect that the broadening of the absorption lines in the supergiants of very early type is not produced by rotation". The question of interest, of course, is not what we call the mechanism, but its physical origin.

\subsection{Stellar winds}

Those stars which show the largest 'turbulence' are also those which have the strongest stellar winds, and there are many examples of line displacements or asymmetries which are clearly attributable to outflows. Simplified models argue that line formation in the transsonic region can be important in determining metal-line profiles (e.g., Kudritzki 1992), and this may often be the dominant factor. However, in the absence of detailed calculations, it remains unclear whether this is the only factor, and there are several other potentially significant 


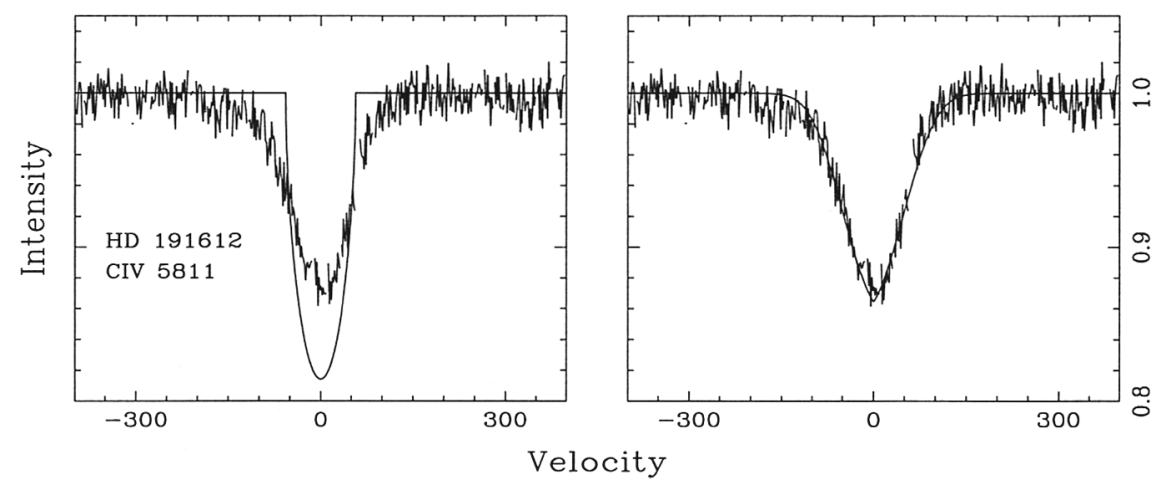

Figure 5. The C IV $\lambda 5811$ line in the spectrum of the O star HD 191612. The model on the left is a pure rotation profile with the same full width at half depth and the same equivalent width as the observed profile; it is a very poor match to the data. The model on the right is dominated by transverse turbulence.

processes which cannot yet be eliminated, particularly in those cases where data of good S:N ratio show no evidence of asymmetries or displacements.

\subsection{Pulsation}

A number of OB stars are known to be non-radial pulsators (see Townsend 2003). Although low-order modes are recognizable spectroscopically or photometrically, a broad spectrum of high-order modes is unlikely to have to have a clear timedependent signature. However, if the velocity fields are of sufficient amplitude, they may nonetheless modify the overall line profile in a recognizable way. Illustrative calculations by R. Townsend, K.C. Smith, and the author confirm this quantitatively, but are handicapped by the absence of a priori predictions on the expected amplitudes and pulsation quantum numbers.

\subsection{Differential Rotation}

Line-profile calculations almost invariably contain the assumption of uniform rotation (i.e., latitude-independent angular velocity at the stellar surface), but differential rotation can easily move absorption from one part of the line profile to another, through simple Doppler shifts. However, no evidence has been found for differential rotation in high-quality spectroscopy of stars with radiative envelopes (e.g., Gray 1977; Howarth \& Smith 2001). Moreover, if rotation is, as proposed by Zahn, 'shellular', then we would expect uniform surface rotation (since the surface is an isobar, or 'shell').

\subsection{Turbulent motions}

By 'turbulent motions' I don't mean turbulence as understood by a fluid hydrodynamicist, but merely the bulk motion of material in the line-forming regions. Turbulence, in the sense of a suprathermal line broadening, was introduced by Rosseland (1928), as a desaturating mechanism; we now call this 'microturbulence'. The turbulent cell size in this case is supposed to be small compared to the mean free path of the line photon, and microturbulence is implemented in 
practice by increasing the thermal width in the atomic absorption coefficient; it therefore naturally takes on a gaussian form (cf. McErlean, Lennon \& Dufton 1998, Smith \& Howarth 1998, Villamariz \& Herrero 2000 for OB-star studies).

'Macroturbulence', by contrast, is supposed to characterize motions with cell sizes which are large compared to the photon mean free path. Unlike microturbulence, macroturbulence does not change the line strength - it just redistributes a given amount of absorption through the line profile. (To first order, rotation can therefore be considered a 'macro' process.)

Yet again, we have no a priori theoretical expectation of how macroturbulent motions should be characterized (if they exist at all in radiative envelopes). The simplest model, scaling up the microturbulent paradigm, is one of spatially isotropic, gaussian turbulence; this has the advantage that its effect can be implemented as a simple gaussian smooth of a model flux spectrum. This model has, pragmatically, been adopted in the handful of published hot-star studies (Ebbets 1979; Smith \& Howarth 1998; Ryans et al. 2002). The result of introducing an additional free parameter is usually a substantially improved match to observations, but unfortunately there is no obvious physical basis for isotropic gaussian turbulence in hot-star atmospheres. The improvements in model fits therefore don't really advance our understanding very much.

\subsection{Tangential turbulence?}

The cool-star community have long pursued a 'radial-tangential' model of turbulence, based on the notion of convective cells in which material rises radially, is transported tangentially, then sinks radially (e.g., Gray 1992). Such models require spatial integrations rather than simple convolutions, but are nonetheless straightforward to conduct.

Preliminary experiments show that, in many cases, the assumption of purely tangential gaussian turbulence provides an excellent match to observations of $\mathrm{O}$ stars (e.g., Fig. 5); the same is not generally true of purely radial gaussian turbulence. Although this at first appears no less an ad hoc 'solution' than isotropic turbulence, it becomes of interest when we recall that Zahn's 1992 study of rotational mixing (central to all modern stellar-evolution calculations incorporating the effects of rotation) predicts highly anisotropic turbulence, with much stronger transport in the horizontal (i.e., 'tangential') direction.

Most probably, much of the non-rotational line broadening which is evident in the spectra of hot, luminous stars does arise through the effects of outflows. However, the rapid acceleration (and concomitant drop in density) of stellar winds above the sonic point suggests that the effects are most likely to be spectroscopically evident on scales on the order of the sound speed $\left(\sim 10 \mathrm{~km} \mathrm{~s}^{-1}\right)$, at least for weak and/or high-excitation lines; while in practice many line profiles are distorted on much larger scales (in observed wavelength). Although as yet we have no sound prescription for the horizontal turbulence which dominates in the Zahn picture, it is nonetheless an interesting speculation, encouraged by provisional modelling, that at least some part of the non-rotational line broadening might be the direct signature of rotationally-induced turbulence.

I thank Rich Townsend, the late Keith Smith, Kaj Siebert, Stan Owocki, and Doug Gies, for trying to enlighten me in one way or another. 


\section{References}

Abt, H.A., Morrell, N.I. 1995, ApJS 99, 135

Abt, H.A., Levato, H., Grosso, M. 2002, ApJ 573, 359

Balona, L. 1974, MNRAS 78, 51

Chauville, J., Zorec, J., et al. 2001, A\&A 378, 861

Collins, G.W. 1974, ApJ 191, 157

Collins, G.W., Truax, R.J. 1995, ApJ 439, 860

Conti, P., Ebbets, D. 1977, ApJ 213, 438

Ebbets, D. 1979, ApJ 227, 510

Fliegner, J., Langer, N., Venn, K.A. 1996, A\&A 308, L13

Gray, D. 1977, ApJ 211, 198

Gray, D. 1992: The observation and analysis of stellar photospheres (CUP)

Halbedel, E.M. 1996, PASP 108, 833

Herrero, A., Kudritzki, R.-P., et al. 1992, A\&A 261, 209

Howarth, I.D., Siebert, K.W., Hussain, G.A.J., Prinja, R.K. 1997, MNRAS 284, 265

Howarth, I.D., Smith, K.C. 2001, MNRAS 327, 353

Kudritzki, R.P. 1992, A\&A 266, 395

Maeder, A. 2003, these proceedings

McErlean, N.D., Lennon, D.J., Dufton, P.L., 1998, A\&A 329, 613

Owocki, S. 2003, these proceedings

Penny, L.R. 1996, ApJ 463, 737

Rosseland, S. 1928, MNRAS 89, 49

Royer, F., et al. 2002, A\&A 381, 105 \& 393, 897

Ryans, R.S.I., Dufton, P.L., et al. 2002, MNRAS 336, 577

Schönberner, D., Herrero, A., et al. 1988, A\&A 197, 208

Slettebak, A. 1956, ApJ 124, 173

Slettebak, A. 1966, ApJ 145, 126

Slettebak, A. 1982, ApJS 50, 55 (S82)

Slettebak, A., Collins, G.W., et al. 1975, ApJS 29, 137 (SC75)

Smith, K.C., Howarth, I.D. 1998, MNRAS 299, 1146

Steele, I.A., Negueruela, I., Clark, J.S. 1999, A\&A 137, 147

Stoeckley, T.R. 1968, MNRAS 140, 141

Struve, O. 1931, ApJ 73, 94

Struve, O. 1952, PASP 64, 117

Townsend, R.H.D. 2003, these proceedings

Venn, K.A. 1999, ApJ 518, 405

Verdugo, E., Talavera, A., Gómez de Castro, A.I. 1999, A\&A 346, 819

Villamariz, M.R., Herrero, A. 2000, A\&A 357, 597

Walborn, N.R. 1976, ApJ 205, 419

Wolff, S.C., Edwards, S., Preston, G.W. 1982, ApJ 252, 322

Zahn, J.-P. 1992, A\&A 265, 115 